Publ. RIMS, Kyoto Univ.

17 (1981), 215-233

\title{
Construction of Transverse Projectable Connections in Some Foliated Bundles
}

$\mathrm{By}$

Haruo SUZUKI*

\section{§ 1. Introduction}

Let $\mathcal{K}$ be a local Lie group [7] and $K \subset \mathcal{K}$ a subgroup such that the left multiplication of every element $k \in K$ on $\mathcal{K}$ is defined. We introduce a notion of partial connections and foliated structures in a bundle $\mathcal{E}$ with the fibre $\mathcal{K}$ and the structure group $K$. Notions of transverse connections and transverse projectable connections $[5,3]$ are generalized to foliated structures in $\mathcal{E}$. Under a certain condition on $\mathcal{K}$, we construct foliated structures and transverse projectable connections in the principal $K$-bundle $E$ associated with $\mathcal{E}$ from those in the Local Lie group bundles.

In consequence of the above fact, construction methods of foliated principal bundles equipped with transverse projectable connections are enlarged and these yield Bott's strong vanishing of characteristic classes for some locally homogeneous $G$-foliation [4]. We assume all manifolds are $C^{\infty}$-manifolds without boundaries, and all maps and bundles are of class $C^{\infty}$.

Theorem 1. 1 (Main theorem). Let $\bar{G}$ be a Lie group and $K \subset \bar{G}$ a compact connected Lie subgroup of $\bar{G}$. Let $\mathfrak{l}$ and $\mathfrak{t}$ be Lie algeberas of $\bar{G}$ and $K$ respectively. Suppose there exists a subalgebra $\phi \subset \mathfrak{l}$ such that

$$
I=f \oplus \phi \quad \text { (direct sum of vector spaces) }
$$

and

Communicated by N. Shimada, February 4, 1980.

" Department of Mathematics, Hokkaido University, Sapporo 060, Japan. 
Let $\mathscr{I}$ be the left coset foliation on $\bar{G} / K$ by the subgroup $G$ of $\bar{G}$ containing $K$. Then the principal G-bundle $p: E=\bar{G} \times{ }_{K} G \rightarrow \bar{G} / K$ has a transverse projectable connection with respect to some lift foliation $\widetilde{\mathscr{I}}_{E}$ of $\mathscr{E}$.

Moreover, in the above theorem if $\Gamma \subset \bar{G}$ is a discrete subgroup operating properly discontinuously and without fixed points on $\bar{G} / K$, then it follows that the principal $G$-bundle $p^{\prime}: E^{\prime}=(\Gamma \backslash \bar{G}) \times_{K} G \rightarrow \Gamma \backslash \bar{G} / K$ $=M$ has a transverse projectable connection with respect to some lift foliation $\widetilde{\mathscr{F}}_{E^{\prime}}$ of the foliation $\mathscr{F}^{\prime}$ on $\Gamma \backslash \bar{G} / K$ induced by $\mathscr{F}$ (Corollary 4.3). Let $q$ be the codimension of $\mathscr{L}^{\prime}$ and $V\left(\mathscr{E}^{\prime}\right)$ the transverse vector bundle of $\mathscr{L}^{\prime}$. Since $V\left(\mathscr{F}^{\prime}\right)$ is associated with the foliated $G$-bundle $p^{\prime}: E^{\prime} \rightarrow M$, we have the Bott's strong vanishing of characteristic classes of $V\left(\mathscr{E}^{\prime}\right)$,

$$
\operatorname{Pont}^{k}\left(V\left(\mathscr{F}^{\prime}\right)\right)=0 \quad k>q,
$$

where Pont ${ }^{k}$ denotes the Pontryagin subgroup in the de Rham cohomology group $H_{D R}^{k}(M)$ (Theorem 4.4).

In Section 2, we construct partial connections in the principal $K$ bundle $E$ from those in the local Lie group bundle $\mathcal{E}$. Section 3 concerns foliated structures and transverse projectable connections in $\mathcal{E}$, and is devoted to construct transverse projectable connections in $E$ from those in $\mathcal{E}$. In Section 4, after reviewing the notion of $G$-foliations and (locally) homogeneous foliations, we prove the main theorem and results obtained by this theorem.

The author wishes to thank the referee for his kind advices.

\section{$\S$ 2. Local Lie Group Bundles}

Let $\mathcal{K}$ be a local Lie group in the sense of L. S. Pontryagin [7, p. 137 and Chapter 10] and $K$ a Lie subgroup of $\mathcal{K}$ such that a left multiplication $K \times \mathcal{K} \rightarrow \mathcal{K}$ of every element of $K$ on $\mathcal{K}$ is defined. Let $e \in K \subset \mathcal{K}$ denote the unit element. Suppose there exists a neighborhood 
$\mathcal{N}$ of $c$ and a left $K$-invariant open set $\widetilde{\mathcal{K}} \subset \mathcal{K}$ such that $c \in \widetilde{\mathcal{K}}$ and a "right multiplication" $\widetilde{K} \times \Re \rightarrow \mathcal{K}$ of every element of $\Re$ on $\widetilde{\mathcal{K}}$ is defined. $\widetilde{K}$ is also a local Lie group containing $K$. Let $\mathcal{C}$ be a fibre bundle over a manifold $M$ with the fibre $\mathcal{K}$ and the structure group $K$. Let $E$ denote the principal $K$-bundle associated with $\varepsilon$. Then we have $\varepsilon=E \times_{K} \mathcal{K}$. We denote projection maps of $\mathcal{E}$ and $E$ by $p_{\varepsilon}$ and $p_{E}$ respectively. We call $\mathcal{E}$ simply a local Lie group bundle.

If we have the right multiplication of $b \in \mathcal{K}$ for $k \in \mathcal{K}$, we define a right multiplication $R_{b}$ for $u=\left[\left(u^{\prime}, k\right)\right] \in \mathcal{E}$ by

$$
R_{b}(u)=u \cdot b=\left[\left(u^{\prime}, k b\right)\right] .
$$

$\widetilde{S}=E \times_{K} \mathcal{K}$ is a fibre subbundle of $c$ and the "right multiplication" $\widetilde{\varepsilon} \times \Re \rightarrow \varepsilon$ of every element of $\boldsymbol{\Omega}$ on $\widetilde{\varepsilon}$ is well defined.

Suppose we have a local Lie group homomorphism $h: \mathcal{K} \rightarrow K$ satisfying the following two conditions:

(i) $\left.h\right|_{\kappa}=\mathrm{id}$.

(ii) If we have $h\left(k_{1}\right)=h\left(k_{2}\right)$ for $k_{1}, k_{2} \in \widetilde{K}$, then there exists $b \in \mathcal{K}$ such that $k_{2}=k_{1} b$.

We call $h$ a canonical homomorphism of $\mathcal{K}$ onto $K$. Obviously we have $h(b)=e$. Let $h_{\mathcal{E}}: \mathcal{E}=(E \times \mathcal{K}) / K \rightarrow E=(E \times K) / K$ be the fibre map induced by the map id $\times h: E \times \mathcal{K} \rightarrow E \times K$.

Lemma 2. 1. If $u \cdot b \in \mathcal{C}^{\prime}$ is defined for $u \in \mathcal{E}$ and $b \in \mathcal{K}$, then we have

$$
h_{\varepsilon}(u \cdot b)=h_{\varepsilon}(u) \cdot h(b) .
$$

Poof. Let $\left(u^{\prime}, k\right) \in E \times \mathcal{K}$ be a representative of $u$. It follows that

$$
\begin{aligned}
h_{\varepsilon}(u \cdot b) & =h_{\varepsilon}\left(\left[\left(u^{\prime}, k\right)\right] \cdot b\right) \\
& =h_{\varepsilon}\left[\left(u^{\prime} \cdot k b\right)\right] \\
& =\left[\left(u^{\prime}, h(k b)\right)\right] \\
& =\left[\left(u^{\prime}, h(k)\right)\right] \cdot h(b) \\
& =h_{\varepsilon}(u) \cdot h(b) .
\end{aligned}
$$

q.e.d.

Let $H$ be a rector subbundle of the tangent bundle $T\left(c^{\prime}\right)$ of the 
local Lie group bundle $\mathcal{E}$, satisfying the following conditions:

(i) $H_{u} \cap \mathcal{K}_{u}=\{0\}$ for every $u \in \mathcal{E}$ and $\mathcal{K}_{u}$ the tangent space to the fibre through $u$.

(ii) If $u \cdot b \in \mathcal{E}$ is defined for $u \in \mathcal{E}$ and $b \in \mathcal{K}$, then we have

$$
H_{u \cdot b}=R_{b^{*}}\left(H_{u}\right) \text {. }
$$

(iii) For every $x \in M$ and $u \in p^{-1}(x)$, the vector subspace $p_{\mathcal{E}^{+}}\left(H_{u}\right)$ of the tangent space $T_{x}(M)$ at $x$ does not depend on the choice of $u$. We call $H$ a partial connection in $\mathcal{E}$. By the condition (iii), we set $F_{x}=p_{\mathcal{E}^{*}}\left(H_{u}\right)$ for $u \in p^{-1}(x)$. We obtain a subbundle structure of $F=\left\{F_{x}\right\}_{x \in M}$ in the tangent bundle $T(M)$ from the subbundle structure of $H \subset T(\varepsilon) . \quad F$ is denoted also by $p_{\varepsilon^{*}}(H)$. If the partial connection $H$ satisfies the following condition, we call $H$ a connection in the local Lie group bundle $\mathcal{E}$ :

(iv) For every $u \in \mathcal{E}$, we have

$$
T_{u}(\mathcal{E})=H_{u} \oplus \mathcal{K}_{u} .
$$

It is noted that (iv) implies (i) and (iii).

Theorem 2.2. If $H$ is a partial connection in $\mathcal{E}$, then there exists a unique partial connection $H^{\prime}$ in the principal K-bundle $E$, associated with $\mathcal{E}$, such that

$$
h_{\mathcal{E}^{*}}\left(H_{u}\right)=H_{h^{(u)}}^{\prime} .
$$

If $H$ is a connection in $\mathcal{E}$, then $H^{\prime}$ is a connection in $E$.

Proof. For every $u \in \widetilde{\mathcal{E}}$, we define $H_{\mathcal{E}^{(u)}}^{\prime}=h_{\mathcal{E}^{*}}\left(H_{u}\right)$. Suppose $h_{\varepsilon}(u)=h_{\varepsilon}(v)$ for $u=\left[\left(u^{\prime}, g\right)\right]$ and $v=\left[\left(v^{\prime}, k\right)\right]$. Since we have $p_{E}\left(u^{\prime}\right)$ $=p_{E}\left(v^{\prime}\right)$, one can assume that $u^{\prime}=v^{\prime}$. Because of the equality $\left[\left(u^{\prime}, h(g)\right)\right]$ $=\left[\left(u^{\prime}, h(k)\right)\right]$, we have $h(g)=h(k)$. By the property (ii) of $h$, there exists $b \in \mathcal{K}$ such that $k=g \cdot b$. It follows that $b \in \operatorname{Ker}(h)$ and

$$
\begin{aligned}
v & =\left[\left(u^{\prime}, k\right)\right]=\left[\left(u^{\prime}, g b\right)\right]=\left[\left(u^{\prime}, g\right)\right] \cdot b \\
& =u \cdot b .
\end{aligned}
$$

By Lemma 2.1, we have $h_{\varepsilon}=h_{\mathcal{E}} \circ R_{b}$ and hence

$$
h_{\mathcal{E}^{+}}\left(H_{v}\right)=h_{\mathcal{E}^{*}}\left(H_{u \cdot v}\right)
$$




$$
\begin{aligned}
& =h_{\mathcal{E}^{+}} \circ R_{b^{\prime}}\left(H_{u}\right) \\
& =h_{\mathcal{E}^{+}}\left(H_{u}\right) .
\end{aligned}
$$

Therefore, if we fix $u^{\prime}=h_{\mathcal{E}}(u)(u \in \widetilde{\mathcal{E}})$, then $H_{u^{\prime}}^{\prime}=h_{\mathcal{E}^{i}}\left(H_{u}\right)$ does not depend on the choice of $u$.

Since we have

$$
E=(E \times K) / K \subset(E \times \widetilde{\mathcal{K}}) / K=\widetilde{\mathcal{E}}
$$

and $\left.h_{\varepsilon}\right|_{E}=\mathrm{id}$,

$$
H_{u^{\prime}}^{\prime}=h_{\mathcal{E}^{+}}\left(H_{u^{\prime}}\right)
$$

is defined for every $u^{\prime} \in E$. By Lemma 2.1, it follows that for every $k \in K$,

$$
\begin{aligned}
H_{u^{\prime} \cdot k}^{\prime} & =h_{\mathcal{E}^{+}}\left(H_{u^{\prime} \cdot k}\right) \\
& =R_{h(k)^{*}} \circ h_{\mathcal{E}^{\prime}}\left(H_{u^{\prime}}\right) \\
& =R_{k^{*}}\left(H_{u^{\prime}}^{\prime}\right) .
\end{aligned}
$$

This is the condition (ii) of partial connection for the family of vector subspaces $H^{\prime}=\left\{H_{u^{\prime}}^{\prime}\right\}_{u^{\prime} \in E}$.

For every $x \in M$ and $u^{\prime} \in p_{E}^{-1}(x)$, we have

$$
\begin{aligned}
p_{E^{*}}\left(H_{u^{\prime}}^{\prime}\right) & =p_{E^{*}}\left(h_{\mathcal{E}^{*}}\left(H_{u^{\prime}}\right)\right) \\
& =p_{\mathcal{E}^{*}}\left(H_{u^{\prime}}\right) .
\end{aligned}
$$

Therefore the condition (iii) of the partial connection for $H$ implies that for $H^{\prime}$.

Since we have

$$
\begin{aligned}
& H_{u^{\prime}} \cap \mathcal{K}_{u^{\prime}}=\{0\}, \\
& \mathcal{K}_{u^{\prime}}=\left(\left.p_{\varepsilon^{+}}\right|_{T_{u^{\prime}}(\varepsilon)}\right)^{-1}(0)
\end{aligned}
$$

for every $u^{\prime} \in E$, we obtain

$$
\begin{aligned}
\operatorname{dim}\left(H_{u^{\prime}}\right) & \geqq \operatorname{dim}\left(H_{u^{\prime}}^{\prime}\right) \\
& \geqq \operatorname{dim}\left(p_{E^{\prime}}\left(H_{u^{\prime}}^{\prime}\right)\right) \\
& =\left(\operatorname{dim}\left(p_{\mathcal{E}^{r}}\left(H_{u^{\prime}}\right)\right)\right. \\
& =\operatorname{dim}\left(H_{u^{\prime}}\right),
\end{aligned}
$$


that is,

$$
\operatorname{dim}\left(H_{u^{\prime}}^{\prime}\right)=\operatorname{dim}\left(p_{E^{*}}\left(H_{u^{\prime}}^{\prime}\right)\right)
$$

and therefore

$$
H_{u^{\prime}}^{\prime} \cap K_{u^{\prime}}=\{0\},
$$

which shows the condition (i) of partial connection for $H^{\prime}$.

Let $\operatorname{dim}\left(H_{u^{\prime}}\right)$ be $r$. A local $r$-frame field for $H$ induces that for $H^{\prime}$ and hence $H^{\prime}$ is a subbundle of $T(E)$. Thus the first part of our theorem is proved.

The condition (iv) of connection for $H$ implies that for $H^{\prime}$ and we obtain the second part.

q.e.d.

\section{§3. Foliated Local Lie Group Bundles and Connections}

Let $\mathcal{E}$ be the local Lie group bundle over a manifold $M$ with fibre $\mathcal{K}$ and structure group $K$ stated in the preceding section. A partial connection $H$ in $\mathcal{E}$ is called flat, if the subbundle $H \subset T(\mathcal{E})$ is involutive. A local Lie group bundle $\mathcal{E}$ is called foliated, if $\mathcal{E}$ is equipped with a flat partial connection. This definition is of course valid for usual principal Lie group bundles. Let $E$ be the principal $K$-bundle associated with $\mathcal{E}$ and $H^{\prime}$ the partial connection in $E$ which is induced by the canonical homomorphism $h$ from $H$ (Theorem 2.2).

Lemma 3. 1. If a partial connection $H$ in $\mathcal{E}$ is flat, then the partial connection $H^{\prime}$ in $E$ is also flat.

Proof. Since the differential map $h_{*}$ preserves commutator operators of vector fields, the subbundle $H^{\prime} \subset T(E)$ is involutive if the subbundle $H \subset T(\mathcal{E})$ is involutive.

q.e.d.

Let $\mathcal{E}$ be foliated by the flat partial connection $H$. Integral submanifolds of the involutive subbundle $H \subset T(\mathcal{E})$ define a foliation on $\mathcal{E}$ which we denote by $\mathscr{E}_{\varepsilon}$. Since the differential map $p_{\mathcal{E}^{*}}$ preserves commutator operators of vector fields, the vector subbundle $F=p_{\varepsilon^{*}}(H) \subset T(M I)$ is also involutive and defines a foliation on $M$ which we denote by 2 . 


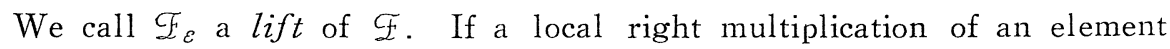
of $\mathcal{K}$ is defined, then it preserves the foliation $\mathscr{E}_{\varepsilon}$. Every leaf $\mathcal{L}_{s}$ of $\mathscr{F}_{\varepsilon}$ is (generally) a "partial covering space" of a leaf of $\mathscr{F}$ under the projection $p_{\varepsilon}$. Conversely, if a foliation $\mathscr{E}_{\varepsilon}$ having properties of lift of a foliation $\mathscr{F}$ on $M$ is given, then $\mathcal{E}$ is foliated through the flat partial connection defined by the vector subbundle $H \subset T(\varepsilon)$ which is tangent to $\mathscr{F}_{\mathcal{E}}$. In this situation, we call $\mathcal{E}$, a foliated local Lie group bundle over a foliated manifold $(M, \Psi)$ and denote it by $\mathcal{E}\left(M, p_{\varepsilon}, \mathcal{K}, \mathcal{F}_{s}\right)$.

Corollary 3.2. Let $h: \mathcal{K} \rightarrow K$ be a canonical homomorphism. If the local Lie group bundle $\mathcal{E}$ with fibre $\mathcal{K}$ and stmucture group $K$ is foliated over a foliated manifold ( $M, \mathcal{F}$ ), then the principal K-bundle $E$ associated with $\mathcal{E}$ has the foliated structure $E\left(M, p_{E}, K, \mathscr{F}_{E}\right)$ over $(M, \mathcal{F})$, induced by $h$.

Proof. Let $H$ be the flat partial connection of the foliated structure of $\mathcal{E}$. By Lemma 3.1, the partial connection $H^{\prime}$ in $E$ determined by $H$ is flat. Since we have $P_{E^{\prime}}\left(H^{\prime}\right)=F, H^{\prime}$ defines a lift foliation $\mathscr{F}_{E}$ of $\mathscr{F}$.

(1.e.d.

Let $\mathcal{H}$ be a connection in the local Lie group bundle $\mathcal{E}$. $\mathcal{H}$ is called adapted to a partial connection $H$ in $\mathcal{E}$, if we have $H_{u} \subset \mathcal{H}_{u}$ for every $u \in \mathcal{E}$. $\mathcal{H}$ is called transverse to the foliation $\mathscr{F}_{\mathcal{E}}$ defined by a flat partial connection $H$ in $\mathcal{E}$, if $\mathscr{H}$ is adapted to $H$. Let $U \subset \mathcal{K}$ be an open neighborhood of $e$. We note that $U$ is again a local Lie group. Let $\hat{\mathcal{E}}$ be a local Lie group bundle over a manifold $N$ with fibre $U$ and $f: \hat{\varepsilon} \rightarrow \mathcal{E}$ an injective fibre map, that is a fibre map which embeds every fibre of $\hat{\mathcal{E}}$ into a fibre of $\mathcal{E}$ and is equivariant with respect to the local right actions of elements in $\mathcal{K}$. For every $u \in \hat{\mathcal{E}}$, we set

$$
\widehat{\mathscr{H}}_{u}=f_{*}^{-1}\left(\mathcal{H}_{f(u)}\right) \cap T_{u}(\widehat{\mathcal{E}}) .
$$

It is easy to see that the family of vector subspaces,

$$
\left\{c \hat{\mathcal{H}}_{u}\right\}_{u}=\hat{\varepsilon}
$$

defines a connection $\mathscr{\mathcal { H }}$ in $\hat{\mathcal{E}}$. We call $\widehat{\mathcal{H}}$ a connection incluced by $f$ 
from $\mathscr{H}$ and denote it also by $\widehat{\mathcal{H}}=f^{*} \mathscr{H}$.

Let $\mathcal{H}$ be a connection in $\mathcal{E}\left(M, p_{\varepsilon}, \mathcal{K}, \mathscr{F}_{\varepsilon}\right)$ which is transverse to $\mathscr{F}_{\varepsilon}$. If for every point of $M$, there is its neighborhood $U$ for local submersion $\phi: U \rightarrow B$ of $\mathscr{E}$ and a subbundle $\mathscr{R}$ of $\left.\mathcal{E}\right|_{U}$ having some open neighborhood of $e$ in $\mathcal{K}$, as fibre, such that in $\mathcal{R}, \mathcal{H}$ is induced by an injective fibre map $f$ covering $\phi$ from a connection in a local Lie group bundle over $B$ with fibre $\mathcal{K}$, then we call projectable. Let $H$ be the flat partial connection defined by $\mathscr{E}_{\varepsilon}$. For every $u \in \mathscr{R}$, we have $H_{u}=\operatorname{ker}\left(\left.f_{*}\right|_{T_{u}(\varepsilon)}\right)$. So $\left.\mathcal{H}\right|_{\mathscr{R}}$ is adapted to $\left.H\right|_{\mathscr{R}}$ and $f$ is a local submersion of $\mathscr{F}_{\varepsilon}$.

Lemma 3. 3. Let $H$ be a partial connection in the local Lie group bundle $\mathcal{E}$ with fibre $\mathcal{K}$ and structure group $K$, and $\mathcal{H}$ a connection in $\mathcal{E}$. Let $E$ be the principal K-bundle associated with $\mathcal{E}$. Let $H^{\prime}$ be the partial connection in $E$ induced by a canonical homomorphism $h: \mathcal{K} \rightarrow K$ from $H$ and $\mathcal{H}^{\prime}$ the connection in $E$ induced by $h$ from $\mathcal{H}$ (Theorem 2.2). If $\mathcal{H}$ is adapted to $H$, then $\mathcal{H}^{\prime}$ is also adapted to $H^{\prime}$.

Proof. Let $h_{\mathcal{E}}: \mathcal{E} \rightarrow E$ be the fibre map induced by the canonical homomorphism $h$. Since for every $u \in \mathcal{E}$ we have $H_{u} \subset \mathcal{H}_{u}$, it follows that for every $u^{\prime} \in E$

$$
H_{u^{\prime}}^{\prime}=h_{\varepsilon^{*}}\left(H_{u^{\prime}}\right) \subset h_{\varepsilon^{*}}\left(\mathcal{H}_{u^{\prime}}\right)=\mathcal{H}_{u^{\prime}}^{\prime} . \quad \text { q.e.d. }
$$

Theorem 3.4. Let $\mathcal{E}=\mathcal{E}\left(M, p_{\varepsilon}, \mathcal{K}, \mathscr{I}_{\varepsilon}\right)$ be a foliated local Lie group bundle over a foliated manifold $(M, \mathscr{F})$ and let $E=E\left(M, p_{E}, K\right.$, $\left.\mathscr{F}_{E}\right)$ be the foliated principal K-bundle over $(M, \mathscr{I})$ induced by a canonical homomorphism $h: \mathcal{K} \rightarrow K$ from $\mathcal{E}\left(M, p_{\varepsilon}, \mathcal{K}, \mathscr{F}_{\varepsilon}\right)$ in the principal $K$-bundle $E$ associated with $\mathcal{E}$ (Corollary 3.2). If a connection $\mathcal{H}$ in $\mathcal{E}$ is transverse to $\mathscr{F}_{\mathcal{E}}$, then the connection $\mathcal{H}^{\prime}$ in $E$ induced from $\mathcal{H}$ is transverse to $\mathscr{F}_{E}$. Moreover, if a transverse connection $\mathcal{H}$ in $\mathcal{E}\left(M, p_{\varepsilon}, \mathcal{K}, \mathscr{F}_{\varepsilon}\right)$ is projectable, then the transverse connection $\mathcal{H}^{\prime}$ in $E\left(M, p_{E}, K, \mathscr{I}_{E}\right)$ induced from $\mathcal{H}$ is also projectable.

Proof. The first part is a direct consequence of Lemma 3.3. We 
prove the second part in the following. Projectability of transverse connection in $\mathcal{E}\left(M, p_{\varepsilon}, \mathcal{K}, \mathscr{F}_{\varepsilon}\right)$ is a local property and hence one can assume without loss of generality that

(i) $\quad M=\boldsymbol{R}^{n}=\boldsymbol{R}^{n-q} \times \boldsymbol{R}^{q}, \quad \mathscr{F}=\left\{\mathbb{R}^{n-q} \times\{a\} \mid a \in \mathbb{R}^{q}\right\}$,

(ii) $\mathcal{E}$ is a trivial bundle $\mathbb{R}^{n} \times \mathcal{K}$ with the natural projection $p_{\mathcal{E}}$ : $\mathbb{R}^{n} \times \mathcal{K} \rightarrow \mathbb{R}^{n}$,

(iii) there exists an open neighborhood $U \subset \mathcal{K}$ of $e$, such that $U \subset \widetilde{\mathcal{K}} \cap \mathcal{N}$ and there exists a map $r: \mathbb{R}^{n}=\mathbb{R}^{n-q} \times \mathbb{R}^{q} \rightarrow U$, such that for every $a \in \mathbb{R}^{q}$ we have $\gamma(0, a)=e \in \mathcal{U}$ and

$$
\begin{aligned}
& L_{\mathcal{E}, a, k}=\left\{(x, a, \gamma(x, a) k) \mid x \in \boldsymbol{R}^{n-q}\right\} \quad\left(a \in \mathbb{R}^{q}, k \in U\right), \\
& \left.\mathscr{I}_{\varepsilon}\right|_{\boldsymbol{R}^{n} \backslash u}=\left\{L_{\mathcal{E}, a, k} \cap \boldsymbol{R}^{n} \times U \mid a \in \boldsymbol{R}^{q}, k \in U\right\} .
\end{aligned}
$$

We define an injective fibre map (actually a local isomorphism) $\Phi: \mathbb{R}^{n-q}$ $\times \boldsymbol{R}^{q} \times \mathcal{U} \rightarrow \boldsymbol{R}^{n-q} \times \boldsymbol{R}^{q} \times \mathcal{K} \quad$ by $\Phi(x, a, k)=\left(x, a, \gamma(x, a)^{-1} k\right) . \quad \Phi\left(L_{\varepsilon, a, k}\right.$ $\left.\cap\left(\boldsymbol{R}^{n-q} \times \boldsymbol{R}^{q} \times \mathcal{U}\right)\right)$ is an open set of $\boldsymbol{R}^{n-q} \times\{(a, k)\}$. Similarly we define a $K$-bundle map (actually an isomorphism) $\Phi^{\prime}: \mathbb{R}^{n-q} \times \mathbb{R}^{q} \times K \rightarrow \mathbb{R}^{n-q} \times \mathbb{R}^{q} \times K$ by $\Phi^{\prime}(x, a, k)=\left(x, a, h(\gamma(x, a))^{-1} k\right)$. Setting $L_{E, a, k}^{\prime}=\{(x, a, h(\gamma(x, a)) k) \mid x$ $\left.\in \mathbb{R}^{n-q}\right\}\left(a \in \boldsymbol{R}^{q}, k \in K\right)$, we have $\mathscr{E}_{E}=\left\{L_{E, a, k}^{\prime} \mid a \in \mathbb{R}^{q}, k \in K\right\}$ and $\Phi^{\prime}\left(L_{E, a, k}^{\prime}\right)$ $=\boldsymbol{R}^{n-q} \times\{(a, k)\}$.

Let $\overline{\mathcal{E}}$ be the trivial bundle $\boldsymbol{R}^{q} \times \mathcal{K}$ with the natural projection $p_{\bar{\varepsilon}}: \boldsymbol{R}^{q} \times \mathcal{K} \rightarrow \boldsymbol{R}^{q}$. Let $f: \boldsymbol{R}^{n-q} \times\left(\boldsymbol{R}^{q} \times \mathcal{K}\right) \rightarrow \boldsymbol{R}^{q} \times \mathcal{K}$ be the natural projection defined by $f(x, a, k)=(a, k)$. Since the transverse connection $\mathcal{H}$ in $\mathcal{E}$ with respect to $\mathscr{F}_{\mathcal{E}}$ is projectable, (replacing $U$ by a smaller one if necessary) one can assume that the connection $\Phi_{*} \mathcal{H}=\left(\Phi^{-1}\right) *\left(\left.\mathscr{H}\right|_{\boldsymbol{R}^{n} \times q}\right)$ in $\Phi\left(\boldsymbol{R}^{n} \times U\right)$ is induced by the injective fibre map $f: \mathbb{R}^{n} \times U=\mathbb{R}^{n-q}$ $\times\left(\boldsymbol{R}^{q} \times \mathcal{U}\right) \rightarrow \boldsymbol{R}^{q} \times \mathcal{K}=\overline{\mathcal{E}}$ from a connection $\overline{\mathcal{H}}$ in $\overline{\mathcal{E}}$. Therefore we have

$$
\begin{aligned}
\Phi_{*}\left(\mathcal{H}_{(x, a, \gamma(x, a))}\right) & =f_{*}^{-1}\left(\overline{\mathcal{H}}_{(a, e)}\right) \cap T_{(x, a, e)}\left(\boldsymbol{R}^{n} \times \mathcal{U}\right) \\
& =T_{x}\left(\boldsymbol{R}^{n-q}\right) \times \overline{\mathcal{H}}_{(a, e)} .
\end{aligned}
$$

The fibre maps $h_{\varepsilon}: \boldsymbol{R}^{n} \times \mathcal{K} \rightarrow \boldsymbol{R}^{n} \times K$ and $h_{\bar{\varepsilon}}: \boldsymbol{R}^{q} \times \mathcal{K} \rightarrow \mathbb{R}^{q} \times K$ are given by $h_{\mathcal{E}}(x, a, k)=(x, a, h(k))$ and $h_{\bar{\varepsilon}}(a, k)=(a, h(k))$ respectively. Let $\breve{h}$ be the Lie algebra homomorphism of $h$. Since we have $h_{\varepsilon} \circ \Phi$ $=\Phi^{\prime} \circ h_{\varepsilon}$, it follows that 


$$
\begin{aligned}
& \Phi_{*}^{\prime}\left(\mathcal{H}_{(x, a, h(\gamma(x, a)))}^{\prime}\right)=\Phi_{*}^{\prime} \circ h_{\mathcal{E}^{\prime}}\left(\mathcal{H}_{(x, a, \gamma(x, a))}\right) \\
& =h_{\mathcal{E}^{+} \circ} \Phi_{*}\left(\mathcal{H}_{(x, a, \gamma(x, a))}\right) \\
& =h_{\mathcal{E}} \cdot\left(f_{*}^{-1}\left(\overline{\mathcal{H}}_{(a, e)}\right) \cap T_{(x, a, e)}\left(\mathbb{R}^{n} \times \mathcal{U}\right)\right) \\
& =(\mathrm{id} \times \mathrm{id} \times \check{h})\left(T_{x}\left(\boldsymbol{R}^{n-q}\right) \times \overline{\mathcal{H}}_{(a, e)}\right) \\
& =T_{x}\left(\boldsymbol{R}^{n-q}\right) \times(\mathrm{id} \times \breve{h}) \overline{\mathcal{H}}_{(a, e)} \\
& =T_{x}\left(\boldsymbol{R}^{n-q}\right) \times h_{\bar{\varepsilon}^{*}}\left(\overline{\mathcal{H}}_{(a, e)}\right) \\
& =T_{x}\left(\boldsymbol{R}^{n-q}\right) \times \overline{\mathcal{H}}^{\prime}{ }_{(a, e)} \\
& =f_{*}^{-1}\left(\overline{\mathscr{H}}^{\prime}{ }_{(a, e)}\right) \cap T_{(x, a, e)}\left(\boldsymbol{R}^{n} \times K\right) \text {. }
\end{aligned}
$$

The maps $\Phi^{\prime}$ and $f$ are compatible with the right multiplication of every element of $K$ on $\boldsymbol{R}^{n} \times K$ and so the last formula implies projectability of the transverse connection $\mathscr{H}^{\prime}$ in $\boldsymbol{R}^{n} \times K$ with respect to the foliation $\mathscr{I}_{E}=\left\{L_{E, a, k}^{\prime} \mid a \in \boldsymbol{R}^{q}, k \in K\right\}$.

q.e.d.

Let $G$ be a Lie group and $K \subset G$ a closed subgroup of $G$. A foliated $K$-bundle $\widehat{E}=\widehat{E}\left(M, p_{\widehat{E}}, K, \mathscr{E}_{\widehat{E}}\right)$ over foliated manifold $(M, \mathscr{F})$ induces uniquely the foliated $G$-bundle structure $E\left(M, p_{E}, G, \mathscr{E}_{E}\right)$ in the principal $G$-bundle $E=\widehat{E} \times{ }_{K} G$ associated with the $K$-bundle $\widehat{E}$, by the right multiplication of an element of $G$. A transverse connection in $\widehat{E}\left(M, p_{\widehat{E}}, K, \mathscr{F}_{\widehat{E}}\right)$ also yields naturally a unique transverse connection in $E\left(M, p_{E}, G, \mathscr{F}_{E}\right)$ by the differential map of the right multiplication of an element of $G$. We close this section by the following Lemma which is proved in a similar way to Theorem 3. 4 and easier than it.

Lemma 3.5. Let $\mathscr{H}$ be the transverse connection in $E\left(M, p_{E}, G, \mathscr{F}_{E}\right)$ which is determined by a transverse connection $\widehat{\mathcal{H}}$ in $\widehat{E}\left(\Lambda, p_{\hat{E}}, K, \mathscr{F}_{\widehat{E}}\right)$. If $\widehat{\mathcal{H}}$ is projectable, then $\mathcal{A}$ is also projectable in $E\left(M, p_{E}, G, \mathscr{F}_{E}\right)$.

Proof. Projectability of a transverse connection in $\widehat{E}=\widehat{E}\left(M, p_{\widehat{E}}, K\right.$, $\left.\mathscr{F}_{\hat{E}}\right)$ is a local property and hence, as in the proof of Theorem 3.4, one can assume that

(i) $\quad M=\boldsymbol{R}^{n}=\boldsymbol{R}^{n-q} \times \boldsymbol{R}^{q}, \quad \mathcal{F}=\left\{\boldsymbol{R}^{n-q} \times\{a\} \mid a \in \boldsymbol{R}^{q}\right\}$,

(ii) $\widehat{E}$ is a trivial bundle $\mathbb{R}^{n} \times K$ with the natural projection 
$p_{\hat{E}}: \mathbb{R}^{n} \times K \rightarrow \mathbb{R}^{n}$,

(iii) there exists a map $\gamma: \mathbb{R}^{n}=\mathbb{R}^{n-q} \times \mathbb{R}^{q} \rightarrow K$ such that $\gamma(0, a)$ $=e \in K \subset G$ (the unit element of $G$ ) for every $a \in \mathbb{R}^{q}$,

$$
L_{\widehat{E}, a, g}=\left\{(x, a, \gamma(x, a) g) \mid x \in \mathbb{R}^{n-q}\right\}
$$

and

$$
\mathscr{F}_{\widehat{E}}=\left\{L_{\widehat{E}, a, g} \mid a \in \mathbb{R}^{q}, \ell_{\ell} \in K\right\} .
$$

We define a $K$-bundle map $\Phi: \widehat{E} \rightarrow \widehat{E}$ by $\Phi(x, a, k)=\left(x, a, \gamma(x, a)^{-1} k\right)$. Since we have $\Phi\left(L_{\widehat{E}, a, k}\right)=\mathbb{R}^{n-q} \times\{(a, k)\}$, (iii) is reduced to

(iii') $\mathscr{I}_{\hat{\boldsymbol{E}}}=\left\{\mathbb{R}^{n-q} \times\{(a, k)\} \mid a \in \mathbb{R}^{q}, k \in K\right\}$.

Let $\widetilde{E}$ be a trivial bundle $\mathbb{R}^{q} \times K$ with the projection $p_{\widetilde{E}}: \mathbb{R}^{q} \times K \rightarrow \mathbb{R}^{q}$. Because of the condition of theorem, one can assume that the connection $\widehat{\mathscr{H}}$ in $\widehat{E}$ is induced from a connection $\widetilde{\mathcal{H}}$ in $\widetilde{E}$, by the submersion of $\mathscr{F} \widehat{E}$, $\widehat{f}: \widehat{E}=\mathbb{R}^{n} \times K=\mathbb{R}^{n-q} \times\left(\mathbb{R}^{q} \times K\right) \rightarrow \widetilde{E}=\mathbb{R}^{q} \times K$ which maps $(x, a, k)$ to $(a, k)$ and gives a $K$-bundle map over the submersion $\mathbb{R}^{n} \rightarrow \mathbb{R}^{q}$ of the foliation F. It follows that

$$
\begin{aligned}
\widehat{\mathcal{H}}_{(x, a, k)} & =\widehat{f}_{*}^{-1}\left(\widetilde{\mathcal{H}}_{(a, k)}\right) \cap T_{(x, a, k)}\left(\mathbb{R}^{n} \times K\right) \\
& =T_{x}\left(\mathbb{R}^{n-q}\right) \times \widetilde{\mathscr{H}}_{(a, k)} .
\end{aligned}
$$

We have immediately $E=\widehat{E} \times{ }_{K} G=\mathbb{R}^{n} \times G$ and $\widetilde{E} \times{ }_{K} G=\mathbb{R}^{q} \times G$ denoted by $\bar{E}$. Let $i: \widehat{E} \rightarrow E$ and $\bar{i}: \widetilde{E} \rightarrow \bar{E}$ be the natural inclusion maps. By (iii'), one obtains $\mathscr{F}_{E}=\left\{\mathbb{R}^{n-q} \times\{(a, g)\} \mid a \in \mathbb{R}^{q}, g \in G\right\}$. The submersion of $\mathscr{E}_{E}$ is the map $f: E=\mathbb{R}^{n} \times G=\mathbb{R}^{n-q} \times\left(\mathbb{R}^{q} \times G\right) \rightarrow \bar{E}=\mathbb{R}^{q} \times G$ which is defined by $f(x, a, g)=(a, g)$ and gives a $G$-bundle map over the submersion $\mathbb{R}^{n} \rightarrow \mathbb{R}^{q}$ of the foliation $\mathscr{E}$. Let $\overline{\mathscr{H}}$ denote the connection in $\bar{E}$ determined by $i_{*} \widetilde{\mathscr{H}}$ through the differential map of right multiplication of $g \in G$ in $E$.

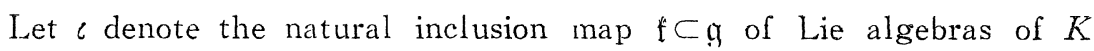
and $G$. By the assumption on $\mathcal{H}$, we have

$$
\begin{aligned}
\mathcal{H}_{(x, a, e)} & =i_{*}\left(\widehat{\mathcal{H}}_{(x, a, e)}\right) \\
& =i_{*}\left(\widehat{f}_{*}^{-1}\left(\widetilde{\mathcal{H}}_{(a, e)}\right) \cap T_{(x, a, e)}\left(\mathbb{R}^{n} \times K\right)\right) \\
& =(\mathrm{id} \times \mathrm{id} \times \iota)\left(T_{x}\left(\mathbb{R}^{n-q}\right) \times \widetilde{\mathcal{H}}_{(a, e)}\right) \\
& =T_{x}\left(\boldsymbol{R}^{n-q}\right) \times(\mathrm{id} \times \iota) \widetilde{\mathcal{H}}_{(a, e)}
\end{aligned}
$$




$$
\begin{aligned}
& =T_{x}\left(\boldsymbol{R}^{n-q}\right) \times \bar{i}_{*}\left(\widetilde{\mathcal{H}}_{(a, e)}\right) \\
& =T_{x}\left(\boldsymbol{R}^{n-q}\right) \times \overline{\mathcal{H}}_{(a, e)} \\
& =f_{*}^{-1}\left(\overline{\mathcal{H}}_{(a, e)}\right) \cap T_{(x, a, e)}\left(\boldsymbol{R}^{n} \times G\right) .
\end{aligned}
$$

Since $f$ is compatible with the right multiplication of every element of $G$ on $\mathbb{R}^{n} \times G$, the last formula implies projectability of the transverse connection $\mathcal{H}$ in $\mathbb{R}^{n} \times G$ with respect to the foliation $\mathscr{F}_{E}$.

q.e.d.

\section{§4. Locally Homogeneous Foliated Bundles}

At beginning of this section, we examine the canonical homomorphism $h$ of a local Lie group onto its subgroup, which is appeared in the previous sections. Let $L$ be a Lie group and $K \subset L$ a compact connected Lie subgroup. Let $\mathfrak{l}$ and $\mathfrak{f}$ be Lie algebras of $L$ and $K$ respectively. Suppose there exists a Lie subalgebra $\phi \subset \mathfrak{l}$ such that

$$
\mathfrak{l}=\mathfrak{f} \oplus \phi \quad \text { (direct sum of vector spaces) }
$$

and

$$
[\mathrm{f}, \phi] \subset \phi .
$$

Let $C$ and $D$ be small open balls around 0 in $\phi$, such that $C \supset D$. We set $\mathscr{C}=\exp (C)$ and $\mathscr{D}=\exp (D)$, and choose $D$ so small that $\mathscr{D} \cdot \mathscr{D} \subset \mathscr{C}$. It is noted that $\mathscr{D}=\mathscr{D}^{-1}$. We set

$$
\mathcal{K}=K \cdot \mathscr{D}=\{k d \mid k \in K, d \in \mathscr{D}\} .
$$

Since $\mathcal{K}$ is an open set in $L$ and hence it is a local Lie group containing $K$. An obvious left multiplication $K \times \mathcal{K} \rightarrow \mathcal{K}$ of every element of $K$ on $\mathcal{K}$ is defined. If we take a sufficiently small open neighborhood $K_{0}$ of $e$ in $K$ and a sufficiently small open neighborhood $\mathscr{D}^{\prime}$ of $e$ in $\mathscr{D}$, then we have $\left(K_{0} \cdot \mathscr{D}^{\prime}\right)^{2} \subset \mathcal{K}$. We set $\Re=K_{0} \cdot \mathscr{D}^{\prime}$ and $\widetilde{\mathcal{K}}=K \cdot \mathscr{D}^{\prime}$. Then $\boldsymbol{N}$ and $\widetilde{K}$ are open sets in $\mathcal{K}$ and hence they are local Lie groups. Moreover, we have $K \subset \widetilde{\mathcal{K}}$ and a right multiplication $\widetilde{\mathcal{K}} \times \Re \rightarrow \mathcal{K}$ of every element of $\boldsymbol{\Omega}$ on $\widetilde{\mathcal{K}}$ is immediately defined.

Lemma 4. 1. One can find a local Lie group homomorphism $h: \mathcal{K} \rightarrow K$ satisfying the following conditions: 
(i) $\left.h\right|_{K}=\mathrm{id}$.

(ii) If we have $h\left(k_{1}\right)=h\left(k_{2}\right)$ for $k_{1}, k_{2} \in \widetilde{\mathcal{K}}$, then there exists $b \in \mathcal{K}$ such that $k_{2}=k_{1} b$.

Therefore, $h$ is a canonical homomorphism of $\mathcal{K}$ onto $K$.

Proof. Since $K$ is a closed subgroup of $L$, one can assume that $K \cap \mathscr{C}=\{e\}$ and hence every element $k \in \mathcal{K}$ is expressed uniquely as $k=g d$ for $g \in K$ and $d \in \mathscr{D}$. Therefore a map $h: \mathcal{K} \rightarrow K$ is defined by

$$
h(g d)=g,
$$

and $h$ satisfies obviously the condition (i). Suppose $h\left(k_{1}\right)=h\left(k_{2}\right)$ for $k_{1}, k_{2} \in \widetilde{\mathcal{K}} \subset \mathcal{K} . \quad k_{i}(i=1,2)$ is uniquely expressed as $k_{i}=g_{i} d_{i}$ for $g_{i} \in K$ and $d_{i} \in \mathscr{D}^{\prime}$. Then we have $g_{1}=h\left(k_{1}\right)=h\left(k_{2}\right)=g_{2}$. One can assume that without loss of generality that $\mathscr{D}^{\prime}=\left(\mathscr{D}^{\prime}\right)^{-1}$ and hence $d_{1}^{-1} d_{2} \in\left(\mathscr{D}^{\prime}\right)^{2} \subset \mathcal{K}$ which we denote by $b$. Therefore, we obtain

$$
\begin{aligned}
k_{2} & =g_{2} d_{2}=g_{1} d_{1} d_{1}^{-1} d_{2}=g_{1} d_{1} b \\
& =k_{1} b .
\end{aligned}
$$

which shows the condition (ii).

We take a small open ball $C^{\prime}$ around 0 in $\phi$ such that $C^{\prime} \subset C$ and $\mathscr{C}^{\prime} \cdot \mathscr{C}^{\prime} \subset \mathscr{C}$ for $\mathscr{C}^{\prime}=\exp \left(C^{\prime}\right)$. If $D$ and hence $\mathscr{D}$ is sufficiently small, then we have $\mathscr{D} \subset \mathscr{C}^{\prime}$, and for any $k \in K$ and $d \in \mathscr{D}$ there exists an element $d^{\prime} \in \mathscr{C}^{\prime}$ such that $d k=k d^{\prime}$ by the condition $[\mathfrak{f}, \phi] \subset \phi$ and compactness of $K$. It follows therefore that $h$ is a homomorphism. q.e.d.

Now, we review briefly the notions of homogeneous and locally homogeneous $G$-foliations by F. W. Kamber and Ph. Tondeur [4]. Let $\mathscr{F}$ be a codimension $q$ foliation on a manifold $M$ and $V(\mathscr{F})$ the transverse vector bundle (normal bundle) of $\mathscr{F}$. The Bott's connection [1] in $V(\mathscr{F})$ defines a flat partial connection in the frame bundle $P(V(\mathscr{F})$ ) of $V(\mathscr{F})$ and hence the principal $G L(q ; \mathbb{R})$-bundle $P(V(\mathscr{F}))$ has a canonical foliated bundle structure $P(V(\mathscr{I}))=P V\left(M, p, G L(q ; \mathbb{R}), \mathscr{F}_{P V}\right)$. Let $G$ be a closed subgroup of $G L(q ; \mathbb{R})$. If there exists a $G$-reduction $E$ of $P(V(\mathscr{F}))$, such that the canonical foliated bundle structure on 
$P(V(\mathscr{F}))$ arises from a foliated bundle structure $E\left(M, p, G, \mathscr{I}_{G}\right)$, then $\mathscr{I}$ is called a $G$-foliation. It is noted that

$$
P(V(\mathscr{I})) \cong E \times{ }_{G} G L(q ; \boldsymbol{R}),
$$

and the requirement in the definition of $G$-foliation is that the canonical lift foliation $\mathscr{E}_{P V}$ on $P(V(\mathscr{F}))$ is the $G L(q ; \boldsymbol{R})$-equivariant foliation which is given by the $G$-equivariant foliation $\mathscr{F}_{E}$ of the foliated bundle structure on $E$. Both foliations $\mathscr{E}_{P V}$ on $P(V(\mathscr{F}))$ and $\mathscr{E}_{E}$ on $E$ project onto the given foliation $\mathscr{F}$ on $M$.

Let $\bar{G}$ be a Lie group and $G \subset \bar{G}$ a Lie subgroup. The foliation of $\bar{G}$ by the (left) cosets of $G$ defines a $G$-foliation with trivial transverse vector bundle $V_{G}$. The $G$-reduction $\widetilde{E}$ of the frame bundle of $V_{G}$ in this case is the trivial $G$-bundle $\bar{G} \times G$ on $G$. The formula

$$
(\bar{g}, g) \cdot g^{\prime}=\left(\bar{g} g^{\prime}, g^{\prime-1} g\right)
$$

for $(\bar{g}, g) \in \bar{G} \times G$ and $g^{\prime} \in G$ defines the diagonal right action of $G$ on $\bar{G} \times G$. The $G$-orbits of this free action define a foliation on $\bar{G} \times G$, which under the projection $\bar{G} \times G \rightarrow \bar{G}$ maps onto the left coset foliation of $\bar{G}$. The vector bundle $V_{G}$ is associated with the trivial $G$-bundle $\bar{G} \times G$ via the adjoint action of $G$ on the quotient $\mathfrak{l} / \mathfrak{g}$ of the Lie algebras $\mathfrak{l}$ and $\mathfrak{g}$ of the Lie groups $\bar{G}$ and $G$ respectively, that is,

$$
V_{G} \cong E \times_{G} l / \mathfrak{g} \text {. }
$$

The Bott's connection in $V_{G}$ is induced from the foliated structure by the $G$-orbits on $\bar{G} \times G$.

Let $K \subset G$ be a subgroup which is closed in $\bar{G}$. The left coset foliation of $\bar{G}$ by $G$ induced a $G$-foliation $\mathscr{F}$ on the homogeneous space $\bar{G} / K$, which is called a homogeneous foliation. The canonical foliated bundle structure in $\bar{G} \times G$ given by $G$-orbits of the diagonal right action is $K$-equivariant and passes to the quotient situation by $K$. The transverse vector bundle of the foliation $\mathscr{F}$ is associated with the principal G-bundle

$$
E=\bar{G} \times_{K} G \rightarrow \bar{G} / K .
$$

(See, e.g., [2].) The canonical foliated bundle structure on $E$ is denoted by $E\left(\bar{G} / K, p, G, \mathscr{I}_{E}\right)$. We note that the homogeneous $G$-foliation $\mathscr{I}$ is 
$\bar{G}$-invariant.

Let $\bar{E}$ be the principal $\bar{G}$-bundle associated with the principal $G$ bundle $E . \bar{E}$ has the canonical foliated bundle structure $\bar{E}\left(\bar{G} / K, \bar{p}, \bar{G}, \mathscr{F}_{\bar{E}}\right)$ over $(\bar{G} / K, \mathscr{E})$ which arises from $E\left(\bar{G} / K, p, G, \mathscr{E}_{E}\right)$. We try to find a transverse projectable connection in $\bar{E}\left(\bar{G} / K, \bar{p}, \bar{G}, \mathscr{F}_{\bar{E}}\right)$.

Lemma 4. 2. The foliated $\bar{G}$-bundle $\bar{E}=\bar{E}\left(\bar{G} / K, \bar{p}, \bar{G}, \mathscr{F}_{\bar{E}}\right)$ has a transverse projectable connection.

Proof. Since $\bar{E}$ is the principal $\bar{G}$-bundle associated with the $G$-bundle $E$, we have

$$
\begin{aligned}
\bar{E} & =E \times_{G} \bar{G} \\
& =\left(\bar{G} \times{ }_{K} G\right) \times{ }_{G} \bar{G} \\
& =\bar{G} \times{ }_{K} \bar{G} .
\end{aligned}
$$

The diagonal right $\bar{G}$-action on $\bar{G} \times \bar{G}$ induces a foliated bundle structure $\bar{E}\left(\bar{G} / K, \bar{p}, \bar{G}, \overline{\mathscr{F}}_{\bar{E}}\right)$ over the trivial one leaf foliation of $\bar{G} / K$. The tangent subbundle $T\left(\overline{\mathscr{F}}_{\bar{E}}\right)$ for $\overline{\mathscr{F}}_{\bar{E}}$ in the tangent bundle $T(\bar{E})$ is invariant under the diagonal right $\bar{G}$-action. By denoting the tangent bundle along the fibre of $\bar{p}: \bar{E} \rightarrow \bar{G} / K$ by $\ell(\bar{E})$, we have $\bar{G}$-invariant splitting of the vector bundle $T(\bar{E})$,

$$
T(\bar{E}) \cong T\left(\overline{\mathscr{F}}_{\bar{E}}\right) \oplus \ell(\bar{E}),
$$

which defines a $\bar{G}$-invariant connection $\theta$ in $\bar{E}$. Since $\mathscr{E}_{E}$ is the (right) $G$-orbit foliation, every leaf of $\mathscr{F}_{\bar{E}}$ is contained in a leaf of $\overline{\mathscr{E}}_{\bar{E}}$ and hence $\theta$ is a transverse connection in the foliated principal $\bar{G}$-bundle $\bar{E}(\bar{G} / K$. $\left.\bar{p}, \bar{G}, \mathscr{F}_{\bar{E}}\right)$ over the foliated manifold $(\bar{G} / K, \mathscr{F})$. $\theta$ is obviously $G$-invariant and therefore it is a transverse projectable connection in $\bar{E}\left(\bar{G} / K \cdot \bar{p}, \bar{G}, \mathscr{I}_{\bar{E}}\right)$.

q.e.d.

Using the above result, we obtain a transverse projectable connection with respect to some foliated $G$-bundle structure on $E$, which is stated in the main theorem.

Proof of Theorem 1.1. Let $\mathcal{K}$ be the local Lie group which is 
given for the Lie group $\bar{G}=L \supset K$ at the beginning of this section. By the assumptions for the subalgebra $\phi \subset \mathfrak{l}$ and Lemma 4.1 , we have the canonical homomorphism $h: \mathcal{K} \rightarrow K$. Let $\mathcal{E} \subset \bar{E}$ be the local Lie group bundle which has $\mathcal{K}$ as fibre and is associated with the quotient $K$-bundle $\widehat{p}: \bar{G} \rightarrow \bar{G} / K$. The foliated bundle structure $\bar{E}\left(\bar{G} / K, \bar{p}, \bar{G}, \mathscr{F}_{\bar{E}}\right)$ in $\bar{E}$ induces a foliated bundle structure $\mathcal{E}\left(\bar{G} / K, p_{\mathcal{E}}, \mathcal{K}, \mathscr{F}_{\mathcal{E}}\right)$ in $\mathcal{E}$ over the foliated manifold $(\bar{G} / K, \mathscr{I})$ and the transverse projectable connection $\theta$ of Lemma 4.2 induces a transverse projectable connection $\theta_{\varepsilon}$ in the foliated local Lie group bundle $\mathcal{E}\left(\bar{G} / K, p_{\varepsilon}, \mathcal{K}, \mathscr{E}_{\varepsilon}\right)$.

By applying Theorem 3.4 to $\mathcal{E}\left(\bar{G} / K, p_{\varepsilon}, \mathcal{K}, \mathscr{F}_{\varepsilon}\right)$ and the canonical homomorphism $h: \mathcal{K} \rightarrow K$, we observe that the principal $K$-bundle $\hat{p}$ : $\bar{G} \rightarrow \bar{G} / K$ has a foliated bundle structure $\bar{G}\left(\bar{G} / K, \widehat{p}, K, \mathscr{F}_{\bar{G}}\right)$ over $(\bar{G} / K, \mathscr{F})$ and $\theta_{\varepsilon}$ determines a transverse projectable connection $\hat{\theta}$ with respect to this foliated bundle structure. The lift foliation $\mathscr{F}_{\bar{G}}$ is extended naturally to a lift foliation $\widetilde{\mathscr{H}_{E}}$ on the extended $G$-bundle $E=\bar{G} \times{ }_{K} G$ of $\widehat{p}: \bar{G} \rightarrow \bar{G} / K$ and $\hat{\theta}$ defines a transverse projectable connection $\omega$ in the foliated $G$-bundle $E\left(\bar{G} / K, p, G, \widetilde{\mathscr{F}}_{E}\right)$ by applying Lemma 3.5 to $\widehat{E}\left(M, p_{\hat{E}}, K, \mathscr{F}_{\widehat{E}}\right)=\bar{G}(\bar{G} / K$, $\left.\widehat{p}, K, \mathscr{I}_{\bar{G}}\right)$ and $\hat{\theta}=\widehat{\mathscr{A}}$.

q.e.d.

Let $\Gamma \subset \bar{G}$ be a discrete subgroup operating properly discontinuously and without fixed point on $\bar{G} / K$. The $\bar{G}$-invariant homogeneous $G$-foliation $\mathscr{F}$ on $\bar{G} / K$ passes to a $G$-foliation $\mathscr{F}^{\prime}$ on the double coset manifold $\Gamma \backslash \bar{G} / K$ which is called a locally homogeneous foliation (cf. [4]). The transverse vector bundle $V\left(\mathscr{F}^{\prime}\right)$ of the foliation $\mathscr{F}^{\prime}$ is associated with the foliated $G$-bundle

$$
p^{\prime}: E^{\prime}=(\Gamma \backslash \bar{G}) \times{ }_{K} G \rightarrow \Gamma \backslash \bar{G} / K .
$$

(See, e.g., [2].) The lift foliation $\widetilde{\mathscr{F}}_{E}$ of $\mathscr{F}$ and the transverse projectable connection $\omega$ of Theorem 1.1 passes to the lift foliation $\widetilde{\mathscr{I}}_{E^{\prime}}$ of $\mathscr{F}^{\prime}$ and the transverse projectable connection $\omega^{\prime}$ with respect to $\widetilde{\mathscr{F}}_{E^{\prime}}$ respectively. Therefore we have the following result.

Corollary 4. 3. Let $\bar{G}, G$ and $K$ be the Lie groups and the Lie subgroups of Theorem 1. 1. Moreover, let $\Gamma \subset \bar{G}$ be a discrete subgroup operaling properly discontinuously and without fixed points on $\bar{G} / K$ 
so that they defines a locally homogeneous G-foliation $\mathcal{E}^{\prime}$ on the manifold $\Gamma \backslash \bar{G} / K$. Then the principal G-bundle $p^{\prime}: E^{\prime} \rightarrow M=\Gamma \backslash \bar{G} / K$ has a transverse projectable connection reith respect to some lift foliation $\widetilde{\mathscr{F}}_{E^{\prime}}$ of $\mathcal{E}^{\prime}$.

In conclusion, one obtains the Bott's strong vanishing theorem for characteristic classes of the locally homogeneous $G$-foliation $F^{\prime}$.

Theorem 4. 4. Let $\bar{G}$ be a Lie group and $K \subset \bar{G}$ a compacl connected Lie subgroup. Let $\mathfrak{l}$ and $\mathfrak{f}$ be Lie algebras of $\bar{G}$ and $K$ respectively. Suppose there exists a subalgebra $\phi \subset \mathbb{1}$ such that

$$
\mathfrak{l}=\mathfrak{f} \oplus \phi \quad \text { and } \quad[\mathfrak{f}, \phi] \subset \phi .
$$

Let $G \subset \bar{G}$ be a Lie subgroup containing $K$ and $\Gamma \subset \bar{G}$ a discrete subgroup operating properly discontinuously and without fixed points on $\bar{G} / K$ so that they define a locally homogeneous $G$-foliation $\mathscr{F}^{\prime}$ on the manifold $M=\Gamma \backslash \bar{G} / K$. Let $q$ be the codimension of $\mathscr{F}^{\prime}$ and $T^{\top}\left(\mathcal{F}^{\prime}\right)$ the transverse vector bundle of $\mathscr{E}^{\prime}$. Then w' have

$$
\operatorname{Pont}^{k}\left(V\left(\mathcal{F}^{\prime}\right)\right)=0, \quad k>q,
$$

where Pont ${ }^{k}$ denotes the Pontryagin subgroup in the de Rham cohomology group $H_{D R}^{k}(M)$.

Proof. Since the vector bundle $T^{\prime}\left(\mathscr{F}^{\prime}\right)$ is associated with the foliated $G$-bundle $p^{\prime}: E^{\prime}=(\Gamma \backslash \bar{G}) \times{ }_{K} G \rightarrow M$, the transverse projectable connection $(1)^{\prime}$ in the foliated bundle $E^{\prime}\left(M, p^{\prime}, G, \widetilde{\mathscr{F}}_{E^{\prime}}\right)$ of Corollary 4.3 (actually the transverse projectable connection $\hat{\theta}^{\prime}$ in $\Gamma \backslash \bar{G}$ with respect to the restriction $\left.\widetilde{\mathscr{I}}_{\Gamma^{\prime} \backslash \bar{G}}=\left.\widetilde{\mathscr{F}}_{E^{\prime}}\right|_{\left(I^{\prime} \backslash \bar{G}\right)}\right)$ defines a transverse projectable connection $F$ in $V\left(\mathscr{F}^{\prime}\right)$. Let $\Omega=\left(\Omega_{i j}\right)$ be the curvature form of the connection $\nabla$. If $f$ is a $G L(q ; \mathbb{R})$-invariant polynomial of degree $r$ on the Lie algebra $\mathfrak{g l}(q ; \mathbb{R})$ of $G L(q ; \mathbb{R})$, then $f(\Omega)$ is a closed $2 r$-form and the de Rham cohomology class $[f(\Omega)]$ of $f(\Omega)$ is by definition an element of $\operatorname{Pont}^{2 r}\left(V\left(\mathscr{I}^{\prime}\right)\right)$. The curvature form $\Omega$ is locally a pull-back of a curvature form of a connection on a y dimensional manifold and hence we have

$$
f(\Omega)=0 \quad 2 r=k>q . \quad \text { q.e.d. }
$$


Remark. The condition of compactness for the Lie subgroup $K \subset \bar{G}$ in Theorem 1.1 and this section is used to obtain a canonical homomorphism $h$ from a local Lie subgroup $\mathcal{K} \subset \bar{G}$ containing $K$ onto $K$. Thus if the existence of $h$ is assumed, then the compactness of $K$ is weakened to the closedness. For instance, if $\bar{G}$ is a semidirect product of closed subgroups $K$ and $\Phi$ which have Lie algebras $\mathfrak{f}$ and $\phi$ respectively, then the results on (locally) homogeneous $G$-foliations in Theorems 1.1 and 4. 4 still hold good.

Example. The Lie group $L(=\overline{\mathrm{G}})$ and its subgroups in this section can be obtained, for instance, from Lie groups which has the real semisimple graded Lie algebra

$$
g=\sum_{-k \leqq p \leqq k} g_{p}
$$

(see, e.g., [8] and [6]) as follows. Let $\mathfrak{f}^{\prime}$ be a subalgebra of $\mathfrak{g}$ which generates a maximal compact subgroup in the adjoint group of $\mathfrak{g}$. Let $\mathfrak{m}^{\prime}$ be the complementary subalgebra for $\mathfrak{f}^{\prime}$ in the Iwasawa's decomposition of $\mathfrak{g}$ and then we have $\mathfrak{g}=\mathfrak{f}^{\prime} \oplus \mathfrak{n}^{\prime}$. Let $L$ denote the Lie group determined by the Lie subalgebra

$$
\mathfrak{l}=\mathfrak{f} \oplus \phi \subset \mathrm{g},
$$

where $\mathfrak{f}=\mathfrak{f}^{\prime} \cap \mathrm{g}_{0}$ and $\phi=\sum_{1 \leqq p \leqq k} \mathfrak{g}_{p}$. Then $\mathfrak{f}$ and $\phi$ are subalgebras of $\mathfrak{l}$ satisfying the condition

$$
[\mathfrak{f}, \phi] \subset \phi .
$$

Let $\psi \subset Y$ be a subalgebra such that

$$
\psi \supset \mathfrak{i} .
$$

We denote by $K, G \subset \bar{G}$, the connected Lie subgroups defined by f, $\psi$ respectively. The subgroup $K$ and $G$ of the Lie group $\bar{G}$ satisfy the conditions in Theorems 1.1 and 4. 4.

\section{References}

[1] Bott, R., Lectures on characteristic classes and foliations, Lecture Notes in Math., 279, Springer-Verlag, Berlin-Heidelberg-New York, 1972, 1-94.

[2] Kamber, F. W. and Tondeur, Ph., Non-trivial characteristic invariants of homogeneous foliated bundles, Ann. Sci. École Norm. Sup. (4), 8 (1975), 433-486. 
[3] Kamber, F. W. and Tondeur, Ph., Foliated bundles and characteristic classes, Lecture Notes in Math.. 493, Springer-Verlag, Berlin-Heidelberg-New York, 1975.

[4] - G-foliations and their characteristic classcs, Bull. Amer. Math. Soc., 84 (1978), 1086-1124.

[5] Molino, P., Classe d'Atiyah d'un feuilletáge et connexions transverses projectables, C. R. Acad. Sci. Paris Ser. A-B, 272 (1971), A779-A781.

[i] Ochiai, T., Geometry associated with semisimple flat homogencous spaces, Trans. Amer. Math. Soc., 152 (1970), 159-193.

[7] Pontryagin, L. S., Topological groups, 2nd Edition, (Translated from the Russian by A. Brown), Gordon and Breach Science Publ. Inc., New York, 1966.

[8] Tanaka, N., On non-degenerate real hypersurfaces, graded Lie algebras and Cartan connection`, Japan. J. Math., 2 (1976), 131-190. 
